\title{
POP: Preoperative Planning and Simulation Software for Total Hip Replacement Surgery
}

\author{
Constantinos Nikou ${ }^{1}$, Branislav Jaramaz ${ }^{1,2}$, Anthony M. DiGioia III ${ }^{1,2}$, \\ Mike Blackwell ${ }^{1}$, Merle E. Romesberg ${ }^{1}$, and Mallory M. Green ${ }^{1}$ \\ 1 Center for Medical Robotics and Computer Assisted Surgery \\ Carnegie Mellon University, Pittsburgh, PA \\ \{costa, mkb, merle, morgang\}@ri.cmu.edu \\ http://www.mrcas.ri.cmu.edu \\ 2 Center for Orthopaedic Research \\ UPMC Shadyside Hospital, Pittsburgh, PA \\ \{branko, digioia\}@cs.cmu.edu \\ http://www.cor.ssh.edu
}

\begin{abstract}
Proper implant placement during total hip replacement (THR) surgery has been shown to reduce short and long term complication including dislocations, accelerated wear, and loosening of the implants. Correct implant orientation is the most important factor in preventing impingement, which is a major cause of dislocation and wear following total hip replacement surgery. However, proper implant orientation is also dependent upon patient-specific factors such as pelvic anatomy bone coverage and level of femoral osteotomy, and can affect leg lengths and offsets. This paper describes a preoperative planner for THR that enables the surgeon to determine the optimal placement of the femoral and acetabular components for THR taking all of these factors into account. Coupled with a computer-assisted clinical system for precise implant positioning, this approach could significantly improve patient outcomes and lower costs.
\end{abstract}

\section{Introduction}

Primary total hip replacement surgery is one of the most successful surgical procedures performed today. Dislocation following total hip replacement (THR) surgery represents a significant cause of early failure, incurring additional medical costs and patient distress in 1 to $5 \%$ of cases [1,2]. Joint replacement can also result in leg length discrepancies which cause problems such as increased stress on the hip joint, abnormal gait, loss in muscle strength, and implant loosening $[3,4]$.

Proper placement of the acetabular and femoral components can lessen the risk of these postoperative complications. One significant cause of dislocation is impingement of prosthetic components, in which the implant neck hits the rim of the acetabular liner and levers the head out of the socket. This impingement can lead not only to dislocation, but also to accelerated wear of the implants (leading further to instability and implant loosening via bone resorption). Another 
dislocation mechanism is bone-to-bone impingement, where the femoral head is levered out of the socket with a bone-on-bone collision acting as a fulcrum [5]. Placement of the implant components relative to their associated bony structures and relative to one another will determine the envelope of impingementfree range of motion. Proper femoral component choice and placement can also help minimize the leg length inequalities that may result after THR.

Traditional methods of planning typically involve matching only implant size to the anatomy using preoperative radiographs and acetate implant templates. These methods can only provide alignment information relative to the radiographic plane, which is often not representative of the functional orientation of the pelvis or femur. Without full three-dimensional location information for the implants and bones, the envelope of impingement-free motion cannot be calculated.

We have developed a comprehensive preoperative planner (POP) for total hip replacement surgery that includes a joint simulator with real-time range of motion analysis. This planner allows the surgeon to preoperatively determine the desired position and orientation of both the acetabular and femoral implants relative to the patient's anatomy. Once these positions and orientations are established, real-time data describing range of motion limited by implant-to-implant and bone-to-bone impingement is available. Because the data is presented in real time, the surgeon can fine-tune the placement of the implants in order to maximize patient range of motion while monitoring other factors such as leg length and offset, implant surface coverage, and the amount of removed bone.

\section{Methods}

The planning process begins with a preoperative CT scan of the patient. This CT data is used throughout the planning process for visualization purposes. From this data, triangular surface models of the pelvis and femur are constructed with an alternate software package. The datapoints in those surfaces remain in CT coordinates.

\subsection{Setup Stage}

In the Setup Stage, information about the upcoming surgical case is requested. This data includes the surgeon's name, which side of the pelvis is being operated on (left or right hip), and the surgeon's approach. This data is used both for future calculation and for optimizing the user interface controls. The relevant patient data volumes (such as CT volumes and surface models) and an implant database are also associated with the current case in this stage.

\subsection{Pelvic Landmark Stage}

The next stage defines the transform from CT coordinates to a pelvic coordinate system, defined by four points on the pelvis: the left and right anterior iliac spine 

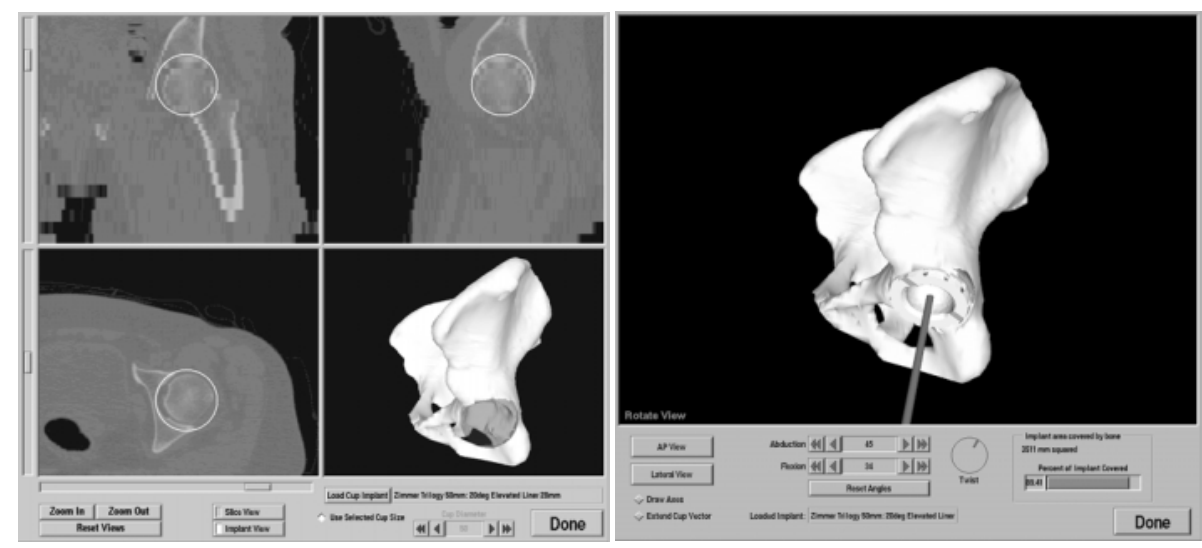

Fig. 1. Acetabular component position and orientation stages.

points and the left and right pubis symphysis points. The iliac spine points and the median of the pubis points define an anterior plane of the pelvis. The pelvic coordinate system lies in this plane, centered at the median of the spine points.

At first, the four pelvic landmark points are chosen automatically by the planner. Their locations are presented to the surgeon in a combined $\mathrm{CT} /$ surface model interface in succession. Coronal, sagittal, and transverse CT slices through each landmark point are presented to the surgeon. The point is marked with cross-hairs on the CT slices as well as by a small sphere on the surface model. This allows the surgeon to view the cross-sections as well as the whole pelvis. The surgeon can change the position of the point with a mouse by clicking on the desired CT slice point or the surface model. Once the surgeon has determined the location of all four points, the points are used to define the anterior pelvic plane and the pelvic coordinate system.

\subsection{Cup Find Stage}

The Cup Find stage presents the surgeon with a surface model of the pelvis. By clicking on a smooth point within the acetabulum, the software can automatically detect the acetabular rim and other points within the acetabulum. These rim points are fit to a plane, which provides an estimate for the orientation of the natural acetabular opening. This orientation is then used as a starting point for placement of the acetabular implant. The size and location of the acetabulum are determined by fitting the points on the surface of the natural cup to a sphere.

\subsection{Cup Position Stage}

Once the location and size of the natural cup is established, POP can then recommend the most appropriately sized implant from the implants available in the database. The surgeon is presented with data similar to the Pelvic Landmark 
stage. This time, however, the cup size and position are marked in the CT slices with a circle. The surgeon can arbitrarily size this circle, or he can fix the circle's size to that of a selected implant. The surgeon can therefore try different implant sizes and styles to determine which implant is the most appropriate for the patient. The surgeon can also use this view to plan the reaming of the acetabulum, determining the level of bone removal required for the given implant size. A sphere in the surface model reflects the selected position and size of the implant cup.

\subsection{Cup Orientation Stage}

The Cup Orientation stage presents the surgeon again with the pelvic surface model along with a surface model of the selected implant. The plastic liner (if relevant) is also displayed. The surgeon is also presented with controls allowing him or her to modify the abduction, flexion (both relative to the pelvic frame), and the twist around the axis of the cup. The surgeon can also orient the cup by dragging the cup's axis about in the window containing the displayed surface model. Bone coverage surface area and percentages are displayed and updated in real time.

\subsection{Femoral Landmark Stage}

In order to plan femoral component placement, a femoral coordinate system must be established. Given the cup location as a starting point, the surgeon locates the femoral head with a similar interface. Once the surgeon accepts the femoral head location, POP then automatically finds other femoral landmark points. These points are the posterior lesser trochanter point, the posterior lateral and medial condyle points, and two points marking the central axis of the femoral canal. The surgeon again refines the location of these points, and moves to the next stage. These points define a femoral coordinate frame parallel to both the mechanical axis of the femur and the plane containing the points on the lesser trochanter and the condyles.

\subsection{Femoral Component Placement Stage}

Finally, the surgeon plans the position and orientation of the femoral component. POP uses relevant information about the chosen acetabular component (e.g. compatible head diameter) along with femoral information (e.g. average thickness of the femur) to choose an appropriate femoral stem/head combination. The femoral landmarks are then used by POP to provide an initial guess for position and orientation of the femoral stem within the bone. The stem is initially placed to match the version of the native femoral neck, aligning its long axis with the axis of the femoral canal. The surgeon is again presented with the three-slice view of the CT along with the surface model. This time, however, the projection of the femoral stem in each slice is shown in the CT views, and 


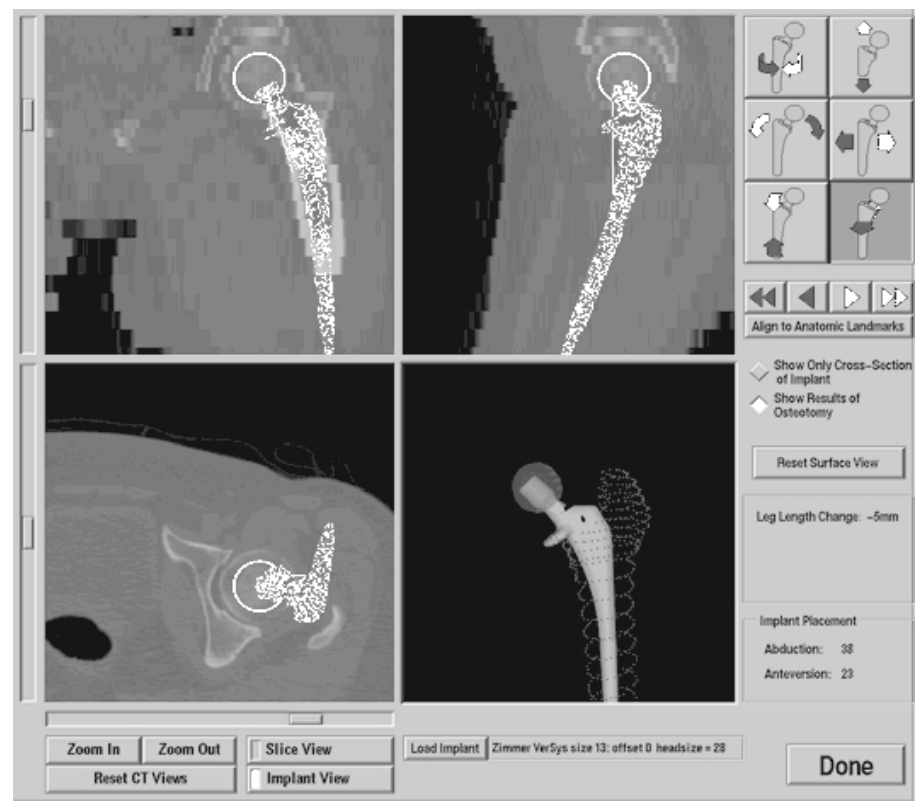

Fig. 2. Femoral component placement stage.

a surface model of the femoral stem is shown along with the femoral surface, which is represented by a point cloud. The surgeon has the option for displaying the femur after the recommended osteotomy associated with the current femoral stem.

The surgeon can re-position the stem with the mouse by clicking on the CT slices or by using buttons that are attached to incremental movements of the stem in 6 degrees of freedom. The CT views of the stem can also be set to display only the proper cross-section of the femoral stem in each plane, allowing the surgeon to determine proper fit of the implant. The surgeon can choose different implants from the implant database, and all information will be updated in real time, including osteotomy calculations, and leg length and offset changes.

\subsection{Range of Motion Analysis Stage}

The final stage, (known as ROM, for Range of Motion) allows the surgeon to check motion limits for arbitrary sets of commonly accepted leg motion. In the ROM stage, the surgeon can determine the allowable envelope of motion for any given placement of implant components, and see the effects of changes in placement in real time. The ROM analysis software here has the same capabilities as in previously published work [7], and has the added feature of bone impingement checking. Bone collision detection is implemented using a point-to-point proximity checking algorithm. For each point in the femoral model at a given position, ROM calculates the distance from that point to all points on the pelvic surface 


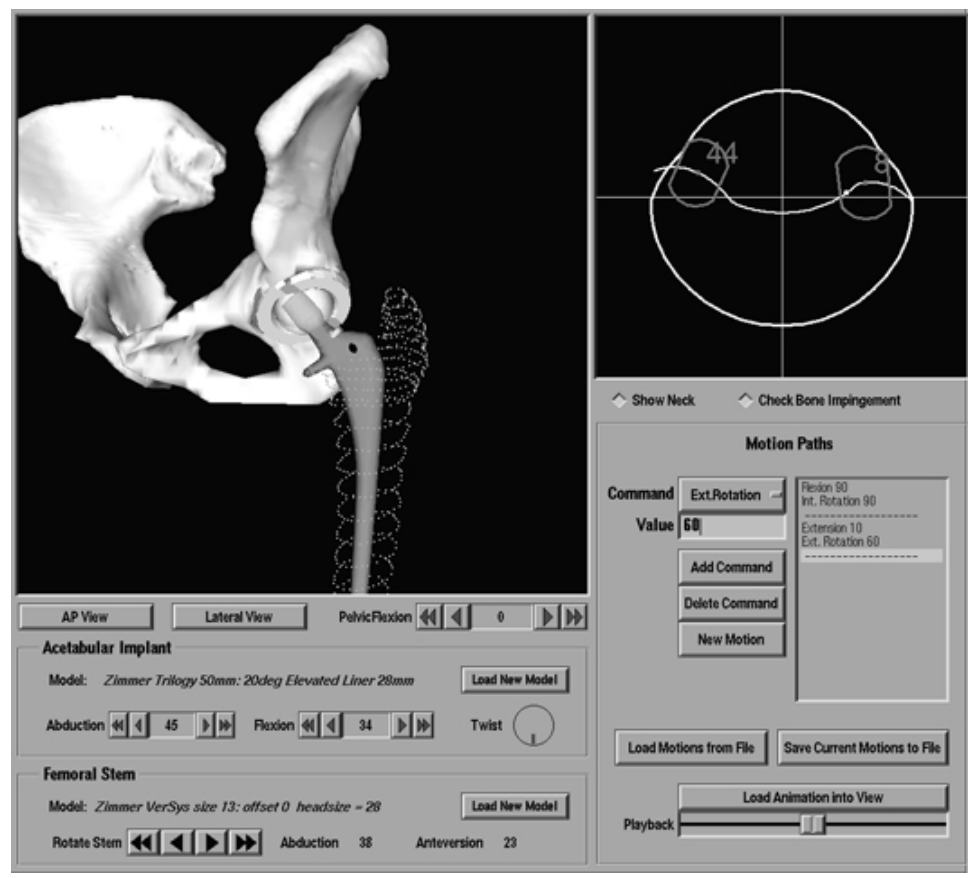

Fig. 3. POP screenshot during ROM simulation stage. In the upper left is a rendered model of the anatomy and implants in their planned orientation. Points of impingement are marked on the models and animations of simulated motions can be viewed. To the upper right is a plot describing impingement limits.

model that lie at a similar distance to the center of the joint. If any of these distances falls below the resolution of the models, then collision is reported. This method of collision detection is fast enough for real-time calculation while providing a conservative estimate for bony impingement. Therefore, testing remains interactive even while determining bone impingement limits, and the surgeon can see instant results of changes in implant cup and liner orientation, femoral stem version, and varied pelvic flexion.

\section{Results}

POP has been used in its current and previous forms for over 50 HipNav cases. Detection of bony impingement is a new feature of the range of motion simulator, and it has proven to be a valuable capability. Without bony impingement checking, incorrect conclusions about range of motion change are possible. To illustrate the effects of simulation, we list the results of a typical case below.

With bone impingement disabled during the planning of one case, the acetabular implant cup was oriented to match the natural opening of the acetabulum (at $45^{\circ}$ abduction $/ 34^{\circ}$ flexion). The femoral implant neck was placed 

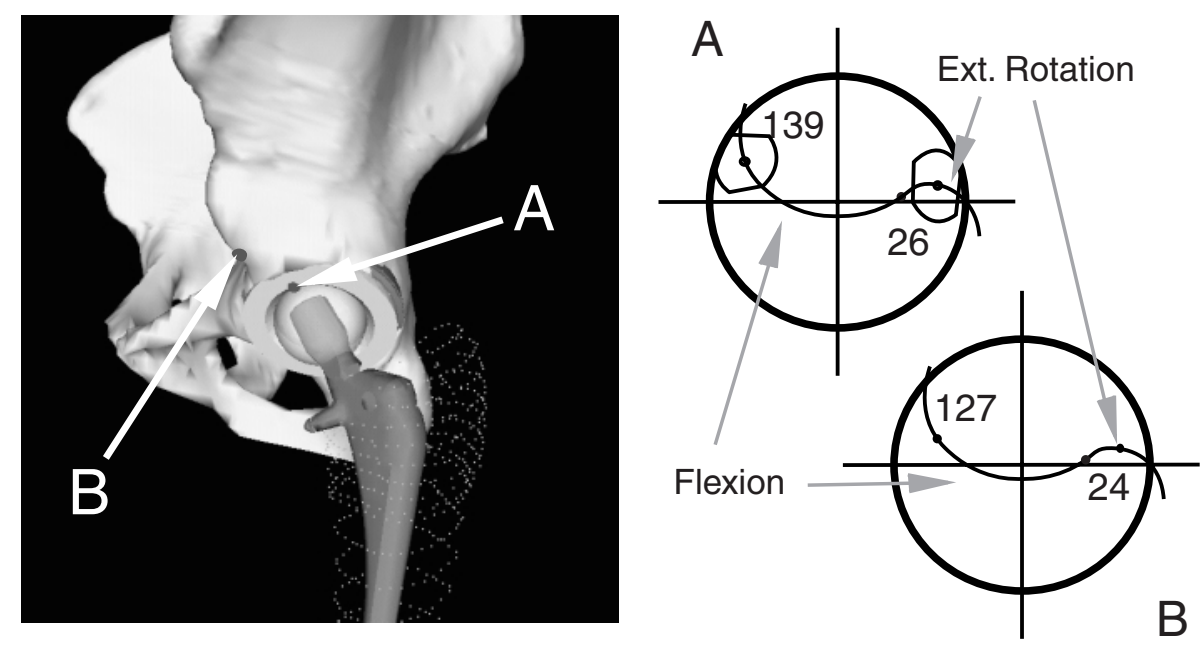

Fig. 4. POP display along with ROM plots for implant (A) vs. bone (B) impingement. The points of impingement on the surface model are impingements during leg flexion. Limits for flexion are $130^{\circ}$ and $112^{\circ}$ and limits for external rotation after extension are $39^{\circ}$ and $36^{\circ}$ for implant and bone impingement respectively

with $37^{\circ}$ abduction, $13^{\circ}$ anteversion, and a $0 \mathrm{~mm}$ functional leg length change. Disregarding bone impingement, the maximal flexion of the femur from neutral position was $130^{\circ}$, and after 10 degrees of extension from neutral position, maximal external rotation was $39^{\circ}$. However, once bone impingement checking was enabled, both the anterior and posterior motion limits were decreased. Possible maximal flexion before bone-to-bone impingement was reduced to $112^{\circ}$, and maximal external rotation after extension was reduced to $36^{\circ}$ (Figure 4 ). Results such as this strengthen previous theories [6] that position of the prosthetic joint components relative to the pelvis within the patient cannot be ignored.

\section{Discussion and Future Work}

POP allows the surgeon to see the effects of implant component placement prior to the surgery, giving the surgeon the chance to optimally plan the procedure. Range of motion analysis and calculation of leg length and offset changes should prove invaluable feature of the system. Though POP is currently being used with the HipNav system, the utility of POP as either a patient-specific planning tool or an educational program is enough to warrant its use without coupling it with an image-guided surgical system.

Though POP has been used with many cases, its use has been limited to one surgeon. Future success of the system will require some optimization of the user-interface, and that is best achieved by getting feedback from a wider user base. Use of this system by a number of surgeons will provide this feedback and 
allow proper studies of patient outcomes for planned cases, provided that the plans can be implemented accurately during surgery.

Future development of POP may include planning for other procedures, such as periacetabular osteotomies and total knee replacement. A software architecture for preoperative planning can be realized, allowing creation of individual planners for separate surgical procedures and creation of planners that are more general, supporting many types of surgery.

\section{References}

1. Cobb, T. K., Morrey B. F., Ilstrup D. M.: The elevated-rim acetabular liner in total hip arthroplasty: Relationship to postoperative dislocation. The Journal of Bone and Joint Surgery, Vol 78-A, No. 1, January 1996, 80-86. 868

2. McCollum, D. E. and Gray, W. J.: Dislocation after total hip arthroplasty (causes and prevention). Clinical Orthopaedics and Related Research 261 (1990): 159-170. 868

3. Hiokka, V., T. Paavilainen, T. Lindholm, T. S., Turula, K. B., and Ylikoski, M.: Measurement and Restoration of Equality in Length of the Lower Limbs in Total Hip Replacement. Journal of Skeletal Radiology (1987) 16: 442-446. 868

4. Woolson, S. T.: Leg Length Equalization During Total Hip Replacement. Orthopedics, January 1990, 13(1): 17-21. 868

5. Amstutz, H. C. and Markolf, K. L.: Design features in total hip replacement. In Harris W.H. (ed.): Proceedings of the Second Open Scientific Meeting of the Hip Society, New York, C.V. Mosby, 1974. 869

6. DiGioia et al.: Image Guided Navigation System to Measure Intraoperatively Acetabular Implant Alignment. Clinical Orthopaedics and Related Research 355 (1998): 8-23. 874

7. Nikou, C., Jaramaz, B., and DiGioia, A.M.: Range of Motion After Total Hip Arthroplasty: Simulation of Non-axisymmetric Implants. Proceedings of the First International Conference on Medical Image Computing and Computer-Assisted Intervention (MICCAI '98), October 1998: 701-709. 872 